Handbuch Gestaltung digitaler und vernetzter Arbeitswelten 
Günter W. Maier - Gregor Engels Eckhard Steffen

Hrsg.

\section{Handbuch Gestaltung digitaler und vernetzter Arbeitswelten}

mit 61 Abbildungen und 12 Tabellen

自 Springer 
Hrsg.

Günter W. Maier

Arbeitseinheit Arbeits- und

Organisationspsychologie, Abteilung

Psychologie \& Research Institute for

Cognition and Robotics (CoR-Lab)

Universität Bielefeld

Bielefeld, Deutschland

Eckhard Steffen

Universität Paderborn

Paderborn, Deutschland
Gregor Engels

Universität Paderborn

Paderborn, Deutschland

ISBN 978-3-662-52898-3

ISBN 978-3-662-52979-9 (eBook)

ISBN 978-3-662-52978-2 (print and electronic bundle)

https://doi.org/10.1007/978-3-662-52979-9

Die Deutsche Nationalbibliothek verzeichnet diese Publikation in der Deutschen Nationalbibliografie; detaillierte bibliografische Daten sind im Internet über http://dnb.d-nb.de abrufbar.

\section{Springer \\ (C) Springer-Verlag GmbH Deutschland, ein Teil von Springer Nature 2020}

Das Werk einschließlich aller seiner Teile ist urheberrechtlich geschützt. Jede Verwertung, die nicht ausdrücklich vom Urheberrechtsgesetz zugelassen ist, bedarf der vorherigen Zustimmung des Verlags. Das gilt insbesondere für Vervielfältigungen, Bearbeitungen, Übersetzungen, Mikroverfilmungen und die Einspeicherung und Verarbeitung in elektronischen Systemen.

Die Wiedergabe von allgemein beschreibenden Bezeichnungen, Marken, Unternehmensnamen etc. in diesem Werk bedeutet nicht, dass diese frei durch jedermann benutzt werden dürfen. Die Berechtigung zur Benutzung unterliegt, auch ohne gesonderten Hinweis hierzu, den Regeln des Markenrechts. Die Rechte des jeweiligen Zeicheninhabers sind zu beachten.

Der Verlag, die Autoren und die Herausgeber gehen davon aus, dass die Angaben und Informationen in diesem Werk zum Zeitpunkt der Veröffentlichung vollständig und korrekt sind. Weder der Verlag, noch die Autoren oder die Herausgeber übernehmen, ausdrücklich oder implizit, Gewähr für den Inhalt des Werkes, etwaige Fehler oder Äußerungen. Der Verlag bleibt im Hinblick auf geografische Zuordnungen und Gebietsbezeichnungen in veröffentlichten Karten und Institutionsadressen neutral.

Springer ist ein Imprint der eingetragenen Gesellschaft Springer-Verlag GmbH, DE und ist ein Teil von Springer Nature.

Die Anschrift der Gesellschaft ist: Heidelberger Platz 3, 14197 Berlin, Germany 


\section{Inhaltsverzeichnis}

Teil I Einführung $\ldots \ldots \ldots \ldots \ldots \ldots \ldots \ldots \ldots \ldots \ldots \ldots \ldots, 1$

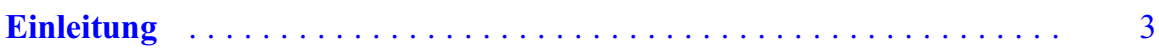

Günter W. Maier, Gregor Engels und Eckhard Steffen

Teil II Gestaltung der digitalisierten Arbeit $\ldots \ldots \ldots \ldots \ldots$

Sicherheit und Gesundheit in der digitalen Arbeitswelt $\ldots \ldots \ldots \ldots 21$

Lars Adolph, Britta Kirchhoff und Jan-Hendrik Geilen

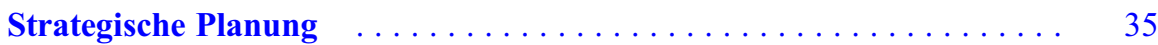

Jürgen Gausemeier, Daniel Eckelt und Christian Dülme

Wie digitale Geschäftsprozesse und Geschäftsmodelle die Arbeitswelt

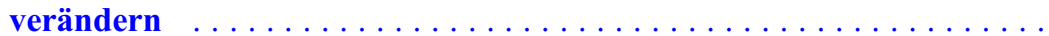

Christoph Plass

Psychologische Arbeitsgestaltung digitaler Arbeitswelten . . . . . . . 87

Lisa Mlekus, Sonja K. Ötting und Günter W. Maier

Digitalisierte Arbeit: Arbeitsrechtliche Aspekte . . . . . . . . . . . 113

Oliver Ricken

Industrial Security by Design $\ldots \ldots \ldots \ldots \ldots \ldots$

Christopher Gerking, Eric Bodden und Wilhelm Schäfer

Technikgestaltung und Ethik für die Arbeitswelt $4.0 \ldots \ldots \ldots$

Klaus Mainzer

Digitale Zukunft: ein inter- und transdisziplinäres Thema . . . . . . . 189

Dagmar Simon und Eckhard Steffen 
Teil III Umsetzung der digitalisierten Arbeit $\ldots \ldots \ldots \ldots \ldots . \quad 201$

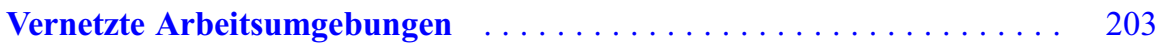

Timm Hörmann und Ulrich Rückert

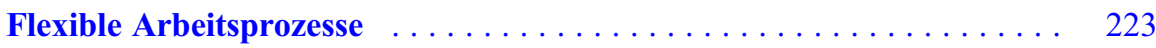

Gregor Engels und Alexander Teetz

Mitbestimmung 4.0: Die digitale Arbeit menschenwürdig gestalten . . 245

Wolfgang Nettelstroth und Gabi Schilling

Wissensmanagement unter Bedingungen von Arbeit $4.0 \ldots \ldots \ldots 7$

Christian Harteis und Christoph Fischer

Betriebsorganisation $\ldots \ldots \ldots \ldots \ldots \ldots \ldots \ldots \ldots \ldots$

Martin Schneider, Christian Manfred Wilke und Anja Iseke

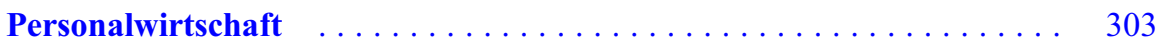

Martin Schneider und Simon Eisele

Kollaborative Roboter: universale Werkzeuge in der digitalisierten

und vernetzten Arbeitswelt

Jochen J. Steil und Günter W. Maier

Change Management für die Einführung digitaler Arbeitswelten

Katharina D. Schlicher, Agnieszka Paruzel, Barbara Steinmann und

Günter W. Maier

Produktentstehung im Zeitalter von Industrie 4.0

Iris Gräßler und Alexander Pöhler

Systems Engineering als Grundlage der Gestaltung digitaler

Arbeitswelten in der Produktentstehung . . . . . . . . . . . . . 405

Roman Dumitrescu, Christian Tschirner und Michael Bansmann

Teil IV Folgen der digitalisierten Arbeit $\ldots \ldots \ldots \ldots \ldots \ldots$

Entwicklung der Beschäftigungsstruktur durch Digitalisierung von

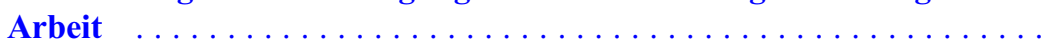

Martin Diewald, Björn Andernach und Eva Susanna Kunze

Digitalisierte Arbeit und private Lebensführung

Martin Diewald, Eva Susanna Kunze und Björn Andernach

Assistierende Technologie zur Förderung beruflichen

Entwicklungspotenzials

Kai Essig, Benjamin Strenge und Thomas Schack 


\section{Über die Herausgeber}

Prof. Dr. Günter W. Maier leitet den Lehrstuhl für Arbeits- und Organisationspsychologie an der Fakultät für Psychologie und Sportwissenschaft der Universität Bielefeld. Er ist stellvertretender Sprecher des NRW Forschungskollegs „Gestaltung von flexiblen Arbeitswelten - Menschenzentrierte Nutzung von Cyber-Physical Systems in Industrie 4.0“ und des NRW Forschungsschwerpunkts „Digitale Zukunft“. Er koordiniert das Themenfeld „Technik und Gesellschaft“ am CoR-Lab der Universität Bielefeld, einem führenden transdisziplinären Kompetenzzentrum für anwendungsorientierte Grundlagenforschung und industriellen Innovationstransfer in den Bereichen Kognition und Robotik.

Prof. Dr. Gregor Engels leitet den Lehrstuhl für Datenbanken und Informationssysteme am Institut für Informatik der Universität Paderborn. Er ist Vorstandsvorsitzender des Software Innovation Labs im Software Innovation Campus Paderborn (SICP), der zentralen Technologie-Transfereinrichtung der Universität Paderborn für software-getriebene Innovationen. Er ist Sprecher des NRW Forschungskollegs „Gestaltung von flexiblen Arbeitswelten - Menschenzentrierte Nutzung von Cyber-Physical Systems in Industrie 4.0“ und des NRW Forschungsschwerpunkts „Digitale Zukunft“.

Prof. Dr. Eckhard Steffen ist Geschäftsführer des PACE (Paderborn Center for Advanced Studies) und leitet die Arbeitsgruppe für „Discrete Mathematics/Graph Theory“ am Institut für Mathematik der Universität Paderborn. Er koordiniert das NRW Forschungskolleg „Gestaltung von flexiblen Arbeitswelten - Menschenzentrierte Nutzung von Cyber-Physical Systems in Industrie 4.0" und ist Mitglied des NRW Forschungsschwerpunkts „Digitale Zukunft“. 


\section{Autorenverzeichnis}

Lars Adolph Bundesanstalt für Arbeitsschutz und Arbeitsmedizin (BAuA), Dortmund, Deutschland

Björn Andernach Fakultät für Soziologie, Arbeitsbereich Sozialstruktur und soziale Ungleichheit, Universität Bielefeld, Bielefeld, Deutschland

Michael Bansmann Technischer Assistent Werkleitung Oelde, Miele Werk Oelde, Oelde, Deutschland

Eric Bodden Fachgruppe Softwaretechnik, Fraunhofer IEM \& Universität Paderborn, Heinz Nixdorf Institut, Paderborn, Deutschland

Martin Diewald Fakultät für Soziologie, Arbeitsbereich Sozialstruktur und soziale Ungleichheit, Universität Bielefeld, Bielefeld, Deutschland

Christian Dülme Heinz Nixdorf Institut, Universität Paderborn, Paderborn, Deutschland

Roman Dumitrescu Fraunhofer-Institut für Entwurfstechnik Mechatronik IEM, Paderborn, Deutschland

Daniel Eckelt Heinz Nixdorf Institut, Universität Paderborn, Paderborn, Deutschland

Simon Eisele Fakultät für Wirtschaftswissenschaften, Universität Paderborn, Paderborn, Deutschland

Gregor Engels Fachgruppe Datenbank- und Informationssysteme, Universität Paderborn, Paderborn, Deutschland

Kai Essig Fakultät Kommunikation und Umwelt, Hochschule Rhein-Waal, KampLintfort, Deutschland

Christoph Fischer AG Erziehungswissenschaft mit dem Schwerpunkt Bildungsmanagement und Bildungsforschung in der Weiterbildung, Universität Paderborn, Paderborn, Deutschland 
Jürgen Gausemeier Heinz Nixdorf Institut, Universität Paderborn, Paderborn, Deutschland

Jan-Hendrik Geilen Bundesanstalt für Arbeitsschutz und Arbeitsmedizin (BAuA), Dortmund, Deutschland

Christopher Gerking Institute for Program Structures and Data Organization, Karlsruhe Institute of Technology (KIT), Karlsruhe, Deutschland

Iris Gräßler Fachgruppe Produktentstehung, Universität Paderborn, Paderborn, Deutschland

Christian Harteis AG Erziehungswissenschaft mit dem Schwerpunkt Bildungsmanagement und Bildungsforschung in der Weiterbildung, Universität Paderborn, Paderborn, Deutschland

Timm Hörmann Technische Fakultät, Universität Bielefeld, Bielefeld, Deutschland Anja Iseke Fachbereich Wirtschaftswissenschaften, Technische Hochschule Ostwestfalen-Lippe, Lemgo, Deutschland

Britta Kirchhoff Bundesanstalt für Arbeitsschutz und Arbeitsmedizin (BAuA), Dortmund, Deutschland

Eva Susanna Kunze Fakultät für Soziologie, Arbeitsbereich Sozialstruktur und soziale Ungleichheit, Universität Bielefeld, Bielefeld, Deutschland

Günter W. Maier Arbeitseinheit Arbeits- und Organisationspsychologie, Abteilung Psychologie \& Research Institute for Cognition and Robotics (CoR-Lab), Universität Bielefeld, Bielefeld, Deutschland

Klaus Mainzer Emeritus of Excellence, Technischen Universität München, München, Deutschland

Carl Friedrich von Weizsäcker-Center, Eberhard Karls Universität Tübingen, Tübingen, Deutschland

Lisa Mlekus Abteilung für Psychologie, Arbeitseinheit Arbeits- und Organisationspsychologie \& Research Institute for Cognition and Robotics (CoR-Lab), Universität Bielefeld, Bielefeld, Deutschland

Wolfgang Nettelstroth IG Metall Bezirksleitung Nordrhein-Westfalen, Düsseldorf, Deutschland

Sonja K. Ötting Abteilung für Psychologie, Arbeitseinheit Arbeits- und Organisationspsychologie \& Research Institute for Cognition and Robotics (CoR-Lab), Universität Bielefeld, Bielefeld, Deutschland

Agnieszka Paruzel Abteilung für Psychologie, Arbeitseinheit Arbeits- und Organisationspsychologie, Universität Bielefeld, Bielefeld, Deutschland

Christoph Plass Vorstand UNITY AG, Paderborn, Deutschland 
Alexander Pöhler Fachgruppe Produktentstehung, Universität Paderborn, Paderborn, Deutschland

Oliver Ricken Fakultät für Rechtswissenschaften, Universität Bielefeld, Bielefeld, Deutschland

Ulrich Rückert Technische Fakultät, Universität Bielefeld, Bielefeld, Deutschland Wilhelm Schäfer Universität Paderborn, Paderborn, Deutschland

Thomas Schack Neurokognition und Bewegung - Biomechanik, Abteilung Sportwissenschaft, Universität Bielefeld, Bielefeld, Deutschland

Gabi Schilling IG Metall Bezirksleitung Nordrhein-Westfalen, Düsseldorf, Deutschland

Katharina D. Schlicher Arbeitseinheit Arbeits- und Organisationspsychologie, Abteilung Psychologie \& Research Institute for Cognition and Robotics (CoRLab), Universität Bielefeld, Bielefeld, Deutschland

Martin Schneider Fakultät für Wirtschaftswissenschaften, Universität Paderborn, Paderborn, Deutschland

Dagmar Simon Wissenschaftszentrum Berlin für Sozialforschung gGmbH, Berlin, Deutschland

Eckhard Steffen Paderborn Center for Advanced Studies, Universität Paderborn, Paderborn, Deutschland

Jochen J. Steil Institute for Robotics and Process Control, Technische Universität Braunschweig, Braunschweig, Deutschland

Barbara Steinmann Abteilung für Psychologie, Arbeitseinheit Arbeits- und Organisationspsychologie, Universität Bielefeld, Bielefeld, Deutschland

Benjamin Strenge Neurokognition und Bewegung - Biomechanik, Abteilung Sportwissenschaft, Universität Bielefeld, Bielefeld, Deutschland

Alexander Teetz Fachgruppe Datenbank- und Informationssysteme, Universität Paderborn, Paderborn, Deutschland

Christian Tschirner Geschäftsführer Two Pillars GmbH, Zukunftsmeile 1, Paderborn, Deutschland

Christian Manfred Wilke Fakultät für Wirtschaftswissenschaften, Universität Paderborn, Paderborn, Deutschland 